\title{
Grey Qualitative Modeling and Control Method for Subjective Uncertain Systems
}

\author{
Peng Wang Shu-Jie Li Yan Lv Zong-Hai Chen \\ Department of Automation, University of Science and Technology of China, Hefei 230027, China
}

\begin{abstract}
A new approach to model and control an unknown system using subjective uncertain rules is proposed. This method is established by combining the grey system theory and the qualitative simulation method. The proposed approach mainly contains three steps. In the first step, subjective uncertain rules are accumulated gradually during cognizing the system; the mapping relations between the system inputs and outputs are built and represented using the grey qualitative matrix in the second step; in the third step, the generalized whitening function is defined to realize the transformation between qualitative and quantitative information. Besides the theoretical results, two sets of simulations based on a water level control system are conducted comparatively to demonstrate the effectiveness of the proposed method. The water level expectation is set to be constant in the first set, while it changes in the second set. The simulation results show that the proposed method tracks the water level expectation well. By combining the proposed method with proportional-integral-derivative (PID) or fuzzy logic controller (FLC), it can be concluded that the system can reach the stable state more quickly and the overshoot can also be reduced compared to using PID or FLC alone.
\end{abstract}

Keywords: Uncertain system, qualitative modeling, knowledge representation, subjective uncertainty, grey qualitative method.

\section{Introduction}

The lack of information makes it difficult to build an accurate mathematical model of uncertain systems. Thus, fuzzy logic ${ }^{[1-6]}$, qualitative simulation ${ }^{[7-11]}$, grey system theory ${ }^{[12,13]}$ etc., are proposed to solve the problem. Fuzzy logic based methods are interested in establishing fuzzy relationships between system states $x$ and $y^{[14,15]}$ : If $x$ is $A_{i}$, then $y$ is $B_{i}, \quad i=1,2, \cdots, k$

where $A_{i}$ and $B_{i}$ are fuzzy concepts defined by fuzzy sets, respectively. However, to model and control a system using fuzzy methods, we need fuzzy rules and membership functions to describe behaviors of the system. Usually, we acquire them by adopting experts' experiences or through experiments ${ }^{[15]}$. However, such information cannot always cover the overall properties of the system. Worse still, we may only obtain a small amount of information. The grey system theory ${ }^{[16]}$, proposed by Deng, originally tries to solve such problems. In the grey system theory, by considering the uncertainty, we can represent system states or observations using grey numbers. The whitening function is built in case the representative value of the grey number is needed. By means of the theory, we can use just a small amount of data to model the system. However, the relations between states or observations are usually quantitative rather than qualitative, which is not intuitive to human. On the contrary, the qualitative simulation methods define the system qualitative states using a set of landmarks, and study how to predict the qualitative states based on current

\footnotetext{
Manuscript received August 21, 2012; accepted January 21, 2014

This work was supported by National Natural Science Foundation of China (No. 61075073 and 61375079).

Recommended by Editor-in-Chief Guo-Ping Liu

(C) Institute of Automation, Chinese Academy of Science and Springer-Verlag Berlin Heidelberg 2015
}

qualitative states and the qualitative difference equations of the system. However, singular inference branches will arise if we use only qualitative information. Kuipers ${ }^{[7,8]}$ tried to reduce the branches by adopting quantitative information to extend the landmarks from qualitative symbols to quantitative intervals. In this paper, we are interested in combining the advantages of both the grey system theory and qualitative simulation to model and control a system.

Let us consider a water level control system shown in Fig. 1. Generally, we can obtain $k$ rules between input $x$ and output $y$ through experiments:

$$
\text { If } x \in P_{i} \text {, then } y \in Q_{i}, \quad i=1,2, \cdots, k
$$

where $P_{i}$ and $Q_{i}$ are classical sets on real number domain $X$ and $Y$. The uncertainty of these rules originates from the incomplete cognition of the system, i.e., we have to cognize the system gradually, with a small amount of information each step. This kind of uncertainty is usually caused by personal cognitive capacity. Therefore, it is subjective uncertainty ${ }^{[17]}$, and the corresponding system is a subjective uncertain system. We call them subjective uncertain rules because they are different than fuzzy rules as $P_{i}$ and $Q_{i}$ are classical sets rather than fuzzy sets. Meanwhile, $P_{i}$ and $Q_{i}$ may be different even for the same input and output because of the personal preference.

Inspired by the grey system theory, we try to use only a small number of subjective uncertain rules to model and control systems. Meanwhile, we use the interval boundaries in the subjective uncertain rules as landmarks to partition the states or observations universe, just like what they do in qualitative simulation methods. Accordingly, by combining the grey qualitative simulation methods published earlier ${ }^{[18-20]}$, we propose the grey qualitative modeling and control method for subjective uncertain systems. First, we 
come up with a structure to represent the subjective uncertain information; second, the boundaries of the sets in subjective uncertain rules are used as landmarks to subdivide the related universes. Each subdivision is a qualitative state of the system. Then, we transform the reasoning relations between rules into relations between subdivisions by integrating the rules, namely building a one-to-one mapping between qualitative sates. At last, we use the whitening function defined on each subdivision to transform qualitative information into quantitative information. For the water level control system shown in Fig. 1, the grey qualitative controller (GQC) is built up based on subjective uncertain rules to model and control the system. The GQC mainly contains three parts: the "accumulating" block in Fig. 1 indicates the accumulation of subjective uncertain rules; the "rule integration" block defines how to integrate the rules to model and control the system; the "whitening" block defines the whitening function. In addition, we can also improve the performance of proportional-integral-derivative (PID) or the fuzzy logic controller (FLC) by combining them with the GQC. The schematic diagram is shown in Fig. 1.

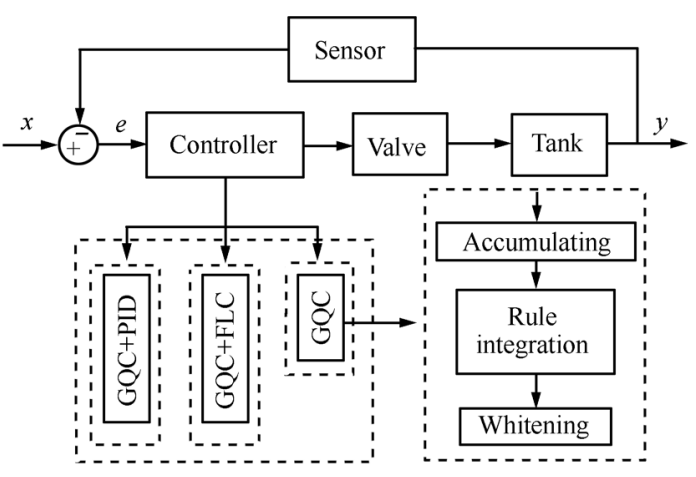

Fig. 1 Subjective uncertain system modeling and control diagram. $x$ is the expectation input level, $y$ is the output, and $e=x-y$. The dashed box at the left bottom contains the controllers we adopt, which will be introduced in detail later. The dashed box at the right bottom shows the main parts of GQC

The remainder of the paper is organized as follows: Section 2 is a short description of the problem to be solved. Section 3 provides details of the grey qualitative modeling and control method we propose. The water level control simulations are carried out in Section 4 to verify the proposed method, and Section 5 concludes our work.

\section{Problem descriptions}

Suppose that we obtain the following subjective uncertain rules after exploring an unknown system:

$$
\text { If } x \in P_{i} \text {, then } y \in Q_{i}, \quad i=1,2, \cdots, k
$$

where $x$ and $y$ are the input and output, respectively, $P_{i}$ and $Q_{i}$ are classical sets on $X$ and $Y$. To simplify the problem, we assume that $P_{i}, Q_{i}$ and $P=\bigcup P_{i}, Q=\bigcup Q_{i}$ are all connected and bounded sets. If they are not, we can convert them into multiple connected sets and analyze them one by one. Besides, suppose that the response function of the system is a one-to-one mapping, i.e., for a given system input $x$, there is only one corresponding output $y$. Based on the assumptions, the problem to be solved includes: 1) Model and control an uncertain system based on subjective uncertain rules; 2) Reduce the uncertainty by integrating subjective uncertain rules. As the uncertain rules are obtained in the cognitive process, conflict rules may arise. For example, suppose that we acquire $P_{i_{1}}, P_{i_{2}}$ and $P_{i_{1}} \cap P_{i_{2}} \neq \varphi$. Thus, if input $x \in P_{i_{1}} \cap P_{i_{2}}$, it is confused to decide which rule to use. Therefore, we should integrate the rules, such that for a given input, we can get a unique output.

\section{Grey qualitative method}

\subsection{Representation of subjective uncer- tain information}

In this subsection, we start with a brief introduction to the grey system theory and the qualitative simulation, and then we give basic concepts related with our new method.

1) Grey system theory

The grey system theory mainly focuses on modeling a system using "small sample" information. Considering the uncertainty, information is usually represented using grey numbers, which are usually intervals. For example, given an observation $y$ of a system output, the grey system theory researchers prefer to represent it using a grey number $[y, \bar{y}]$. It means that the true value of the output is in $[\underline{y}, \bar{y}]$, but we do not know exactly which one it is. To obtain the exact value, a whitening function is usually defined on the grey number to indicate the observer's preference of the values in the interval.

2) Qualitative simulation

In the qualitative simulation, researchers are interested in describing quantities qualitatively. Normally they describe the quantities in terms of their ordinal relations with a small set of landmark values. Brown and De Kleer ${ }^{[21]}$ took the only landmark to be zero, thus the qualitative values were defined as $\{+, 0,-\}$. Kuipers defined a quantity space as a partially ordered set of landmark values, so a quantity was described in terms of its ordinal relations with the landmarks. In the Kuipers' ${ }^{\prime}$ approach, new landmarks are allowed to be discovered and inserted to avoid missing important qualitative distinctions.

Our method inherits the advantages of the two methods, i.e., we try to use a small amount of subjective rules to model the system; meanwhile, new subjective uncertain rules are always accumulated and integrated with the rules already obtained to reduce the uncertainty.

3) Basic concepts in the proposed method

Definition 1. Suppose $P=\left\{p_{i} \mid i=1,2, \cdots, n\right\}$ is a nonempty set and $\otimes(P)$ is the convex hull of $P$. Then we call $\otimes(P)$ a grey number defined on $P$. For clarity, we can denote $\otimes(P)$ as $\otimes$.

Definition 2. If $\otimes$ is a grey number defined on $P$ and 
$B(\otimes)$ indicates the boundary of $\otimes$, then $K=P \bigcap B(\otimes)$ is called the key point set of $\otimes$.

Definition 3. Suppose $S$ is a continuous domain and $q$ is a subjection mapping such that $q: S \rightarrow \tilde{S}, \tilde{S}=\left\{\otimes_{i} \mid i=\right.$ $1,2, \cdots, n\}, \otimes_{i} \cap \otimes_{j}=\phi, i \neq j$. Then $\tilde{S}$ is named a grey qualitative space of $S$, and $\otimes_{i}$ is the grey qualitative element in $\tilde{S}$.

Definition 4. Suppose $R \subseteq \tilde{S} \times \tilde{S}$; then $R$ is called a grey qualitative relationship in $\tilde{S}$.

Definition 5. Suppose $K=\left\{k_{1}, k_{2}, \cdots, k_{m}\right\}$ is the key point set of $\otimes$ and $f: K \rightarrow R$ is a mapping from $K$ to $R$. Then $f\left(k_{1}\right), f\left(k_{2}\right), \cdots, f\left(k_{m}\right)$ are the grey qualitative characteristic values of $\otimes$.

Definition 6. Suppose the grey qualitative characteristic values of $\otimes$ are $f\left(k_{1}\right), f\left(k_{2}\right), \cdots, f\left(k_{m}\right) . \forall p \in \otimes$, we define the generalized whitening function of $\otimes$ as

$$
f(p)=\lambda_{1} f\left(k_{1}\right)+\lambda_{2} f\left(k_{2}\right)+\cdots+\lambda_{m} f\left(k_{m}\right)
$$

where $\lambda_{1}, \lambda_{2}, \cdots, \lambda_{m}$ can be uniquely determined by the relative position of $p$.

The grey qualitative characteristic value is the key quantitative information of the grey qualitative element. By using the generalized whitening function, we can obtain all the quantitative information needed.

In the grey system theory, $f(p)$ indicates the preference of taking $p$ as the representative value of the grey number. In our method, the preference of taking $f(p)$ as the representative value of the grey number is determined by $\lambda_{1}, \lambda_{2}, \cdots, \lambda_{m}$ and $f\left(k_{1}\right), f\left(k_{2}\right), \cdots, f\left(k_{m}\right)$. The meaning of $f(p)$ depends on how $f\left(k_{i}\right), i=1, \cdots, m$ are defined. Thus, the generalized whitening function inherits the idea of the traditional whitening function on the one hand; it expands the meaning of the traditional whitening function on the other hand.

\subsection{Integrating subjective uncertain rules}

Assume that "If $x \in P_{i}$, then $y \in Q_{i}, i=1, \cdots, k$ " are $k$ subjective uncertain rules obtained. Let $a_{i}=\inf P_{i}$, $b_{i}=\sup P_{i}$, where $i=1,2, \cdots, k$, and $a=\inf \bigcup P_{i}$, $b=\sup \bigcup P_{i}$. Meanwhile, let $c_{i}=\inf Q_{i}, d_{i}=\sup Q_{i}$, $i=1,2, \cdots, k$, and $c=\inf \bigcup Q_{i}, d=\sup \bigcup Q_{i}$. Then, $a, a_{i}, b_{i}, b, c, c_{i}, d_{i}, d, i=1, \cdots, k$ can be used as landmarks of $X$ and $Y$. For convenience, we arrange $a, a_{i}, b_{i}, b$ and $c, c_{i}, d_{i}, d, \quad i=1, \cdots, k$ incrementally, resulting in $p_{0}, p_{1}, \cdots, p_{m}$ and $q_{0}, q_{1}, \cdots, q_{n}$, where $p_{0}<p_{1}<\cdots<p_{m}$ and $q_{0}<q_{1}<\cdots<q_{n}$. Let $\otimes_{1}^{i}=\left[p_{i-1}, p_{i}\right)$ (or $\left(p_{i-1}, p_{i}\right]$ ), $i=1,2, \cdots, m$; then $\tilde{S}_{1}=\left\{\otimes_{1}^{i} \mid i=1,2, \cdots, m\right\}$ is the grey qualitative space determined by $p_{0}, p_{1}, \cdots, p_{m}$. Similarly, we call $\tilde{S}_{2}=\left\{\otimes_{2}^{j} \mid j=1,2, \cdots, n\right\}$ the grey qualitative space determined by landmarks $q_{0}, q_{1}, \cdots, q_{n}$.

Based on the process above, we can get the following lemma intuitively.

Lemma 1. $\forall P_{i}, \exists \otimes_{1}^{i_{1}}, \otimes_{1}^{i_{2}}, \cdots \otimes_{1}^{i_{t_{1}}} \in \tilde{S}_{1}$, such that $P_{i}=$ $\bigcup_{k=1}^{t_{1}} \otimes_{1}^{i_{k}} ; \forall Q_{j}, \exists \otimes_{2}^{j_{1}}, \otimes_{2}^{j_{2}}, \cdots \otimes_{2}^{j_{t_{2}}} \in \tilde{S}_{2}$, such that $Q_{j}=$ $\bigcup_{k=1}^{t_{2}} \otimes_{2}^{j_{k}}$.

$R \subset \tilde{S}_{1} \times \tilde{S}_{2}$ is called a grey qualitative reasoning rela- tionship on $\tilde{S}_{1}$ and $\tilde{S}_{2}$, if and only if there exists a subjective uncertain rule "If $x \in \otimes_{1}^{i}$, then $y \in \otimes_{2}^{j}$ ", such that $\left(\otimes_{1}^{i}, \otimes_{2}^{j}\right) \in R$.

Definition 7. Suppose that $R \subset \tilde{S}_{1} \times \tilde{S}_{2}$ is a grey qualitative relation on $\tilde{S}_{1}$ and $\tilde{S}_{2}$. Let

$r_{i j}=\left\{\begin{array}{c}1,\left(\otimes_{1}^{i}, \otimes_{2}^{j}\right) \in R \\ 0,\left(\otimes_{1}^{i}, \otimes_{2}^{j}\right) \notin R\end{array} \quad, i=1,2, \cdots m, j=1,2, \cdots, n\right.$.

Then $R=\left(r_{i j}\right)_{m \times n}$ is called a grey qualitative matrix of $R$, and $R$ is the grey qualitative relation determined by $R=\left(r_{i j}\right)_{m \times n}$.

The integration of subjective uncertain rules is to determine the grey qualitative matrix $R$, with each row sum equals to 1 . It means that for a given subdivision in the input domain, there is only one response subdivision in the output domain. Thus, the second question in Section 2 can be solved.

To prove the existence of $R$, let us begin with constructing the following four row vectors:

1) $A_{i}=\left(a_{i 1}, a_{i 2}, \cdots, a_{i m}\right)$, if $M_{j} \subseteq P_{i}$, then $a_{i j}=1$, otherwise $a_{i j}=0$, where $i=1,2, \cdots, k, j=1,2, \cdots m$.

2) $B_{i}=\left(b_{i 1}, b_{i 2}, \cdots, b_{i n}\right)$, if $N_{j} \subseteq Q_{i}$, then $b_{i j}=1$, otherwise $b_{i j}=0$, where $i=1,2, \cdots, k, j=1,2, \cdots n$.

3) $m_{i}=(0, \cdots, 1, \cdots, 0)$, in which the $i$-th element is 1 , and others are 0 , where $i=1,2, \cdots, m$.

4) $n_{j}=(0, \cdots, 1, \cdots, 0)$, in which the $j$-th element is 1 , and others are 0 , where $j=1,2, \cdots, n$.

"If $x \in P_{i}$, then $y \in Q_{i}$ " means that there exists an $m \times n$ 0-1 matrix $R^{*}$, which satisfies $A_{i} R^{*}=B_{i}$. If the row sum of $R^{*}$ is 1 , and it satisfies the following equation set

$$
\left\{\begin{array}{c}
A_{1} R^{*}=B_{1} \\
A_{2} R^{*}=B_{2} \\
\cdots \\
A_{k} R^{*}=B_{k} .
\end{array}\right.
$$

Then one subdivision in the input domain is related with only one subdivision in the output domain. Therefore, the subjective rules are well integrated. However, the process of acquiring subjective uncertain rules is quite complex, so matrix $R^{*}$ may not be found. To solve the problem, we adopt a weaker equation set shown in (4).

Definition 8. Given two row vectors $A_{i}=$ $\left(a_{i 1}, a_{i 2}, \cdots, a_{i n}\right)$ and $A_{j}=\left(a_{j 1}, a_{j 2}, \cdots, a_{j n}\right)$ with the same dimension, we define $A_{i} \leq A_{j}$ or $A_{j} \geq A_{i}$, if $a_{j r}=1$ can be deduced from $a_{i r}=1, r=1,2, \cdots, n$.

Given the equation set

$$
\left\{\begin{array}{c}
A_{1} R_{0} \leq B_{1} \\
A_{2} R_{0} \leq B_{2} \\
\cdots \\
A_{k} R_{0} \leq B_{k}
\end{array}\right.
$$

where matrix $R_{0}$ is an $m \times n 0-1$ matrix with row sum equals to 1 , we can obtain the following theorem.

Theorem 1. There exists a $0-1$ matrix $R_{0}$, which satisfies (4), with each row sum equals to 1. 
Proof. Let us denote $\tilde{S}_{1}=\left\{\otimes_{1}^{i} \mid i=1,2, \cdots, m\right\}$ as the grey qualitative space of $S_{1}$, and "If $x \in P_{l}$, then $y \in Q_{l}$ " is a known subjective rule.

Suppose that $\exists i$, s.t. $x \in \otimes_{1}^{i}$, and $\otimes_{1}^{i} \subset P_{l}$. Thus "If $x \in \otimes_{1}^{i}$, then $y \in Q_{l}$ " is satisfied.

Based on Lemma 1, we known that $\forall Q_{l}$, $\exists \otimes_{2}^{l_{1}^{q}}, \otimes_{2}^{l_{2}^{q}}, \cdots \otimes_{2}^{l_{t_{2}}^{q}} \in \tilde{S}_{2}$, s.t. $\quad Q_{l}=\bigcup_{k=1}^{t_{2}} \otimes_{2}^{l_{k}^{q}}$. Therefore, "If $x \in \otimes_{1}^{i}$, then $y \in \bigcup_{k=1}^{t_{2}} \otimes_{2}^{l_{k}^{q}}$ " is tenable.

Because the response function of the system is supposed to be a one-to-one mapping, there exists a unique $k_{i}$ satisfying "If $x \in \otimes_{1}^{i}$, then $y \in \otimes_{2}^{l_{k}^{q}}$ ", where $l_{k_{i}}^{q}$ is only relevant to $i$. For clarity, we denote $l_{k_{i}}^{q}$ as $l_{i}^{q}$.

Since $x$ is arbitrarily selected, the aforementioned rules are thus reasonable for all $\otimes_{1}^{i} \in \tilde{S}_{1}, i=1,2, \cdots, m$.

Construct a $0-1$ matrix $R_{0}=\left(r_{i j}\right)_{m \times n}$, where $r_{i, l_{k}^{q}}=1$, $i=1,2, \cdots, m$, while other entries remain zero. Obviously, the row sum of $R_{0}$ is 1 , and we have $m_{i} \cdot R_{0}=n_{l_{i}^{q}}, i=$ $1,2, \cdots, m$.

Based on the definitions of $A_{i}$ and $m_{i}$, we know that if $P_{l}=\bigcup_{k=1}^{t_{1}} \otimes_{1}^{l_{k}^{p}}$, then $A_{l}=\sum_{k=1}^{t_{1}} m_{l_{k}^{p}}$.

Thus, we have $A_{l} R_{0}=\sum_{k=1}^{t_{1}} m_{l_{k}^{p}} R_{0}=\sum_{k=1}^{t_{1}} n_{l_{k}^{q}}$.

Now, we just need to prove $\sum_{k=1}^{t_{1}} n_{l_{k}^{q}} \leq\left(b_{i 1}, b_{i 2}, \cdots, b_{i n}\right)$.

If it is not satisfied, then $\exists \otimes_{1}^{l_{k}^{p}}$, s.t. $x \in \otimes_{1}^{l_{k}^{p}}$ and $y \notin Q_{l}$, but $y \in \bigcup_{k=1}^{t_{2}} \otimes_{2}^{l_{k}^{q}}$. As we already known that $Q_{l}=\bigcup_{k=1}^{t_{2}} \otimes_{2}^{l_{k}^{q}}$, which conflicts with the aforementioned conclusion. Thus, the inequality is sound, and the 0-1 matrix $R_{0}$ satisfies (4).

Based on the aforementioned process, we can obtain the following theorem.

Theorem 2. If $m$ subjective uncertain rules "If $x \in \otimes_{1}^{i}$, then $y \in \otimes_{2}^{l_{i}},(i=1,2, \cdots, m)$ " are tenable, then the rule described in (1) is satisfied.

Definition 9. The subjective uncertain rules defined by $R_{0}$, i.e., "If $x \in \otimes_{1}^{i}$, then $y \in \otimes_{2}^{l_{i}}, i=1,2, \cdots, m$ ", are called subjective uncertain rules integrated from rules represented by (1).

Theorem 1 proves the existence of matrix $R_{0}$, which means that we can find a one-to-one mapping between the input and output subdivisions.

\section{Model of the system}

After the integrating process, we can obtain the following subjective uncertain rules:

If $x \in \otimes_{1}^{i}$, then $y \in \otimes_{2}^{l_{i}}, \quad i=1,2, \cdots, m$.

Based on these rules, we can know that if input $x \in \otimes_{1}^{i}$ then output $y \in \otimes_{2}^{l_{i}}$. However, in real applications, we need a quantitative value to represent the intervals as the traditional controller can only handle single values rather than intervals. In the new modeling and control method, we define the generalized whitening function to transform qualitative information (represented by grey qualitative elements) into quantitative values. The realization of the generalized whitening function depends on the specific problem.
If we know output $y \in \otimes_{2}^{l_{i}}$, we would carefully select $p \in$ $\otimes_{2}^{l_{i}}$ such that $f(p)$ is the maximum value of the generalized whitening function on $\otimes_{2}^{l_{i}}$. Then we will choose $p$ as the representative value of $y$ on $\otimes_{2}^{l_{i}}$. The specific form of the generalized whitening function we use is defined in Section 4.

Based on the analyses above and Fig. 1, the modeling and control procedure of a system of our method mainly contains three steps. 1) Accumulate subjective uncertain rules. Essentially, it is the procedure of transforming the quantitative information into qualitative information. We obtain the subjective rules by searching the grey qualitative elements $\otimes_{1}^{i}$ and $\otimes_{2}^{l_{i}}$ which input $x$ and output $y$ belong to. 2) Integrate the subjective rules: integrate the subjective rules we have obtained and build the grey qualitative matrix $R$ dynamically. 3) Compute the quantitative output using the generalized whitening function. In our method, the output is defined as $y=\arg _{y \in \otimes_{2}^{l_{i}}} \max f(y)$.

\section{Simulation and analysis}

\subsection{Simulation descriptions and settings}

Simulation-based performance analysis of the water level control system shown in Fig. 2 is provided to verify the method proposed in the paper. We assume that the corresponding real system consists of a tank, a liquid level sensor, two valves controlling water flows in and out of the tank, and a controller. Fig. 3 shows the Simulink (Version 2009a) simulation model of the system. Details of the blocks can be found from the "sltank" model in Simulink. We compare our method with both PID and FLC. Nevertheless, we have to proclaim that due to the limits of the cognition capacity, we may not collect enough subjective uncertain rules. Thus, the performance of GQC may not always be as good as PID or FLC. However, the control errors or overshoots etc. can be significantly reduced by combining GQC with PID or FLC.

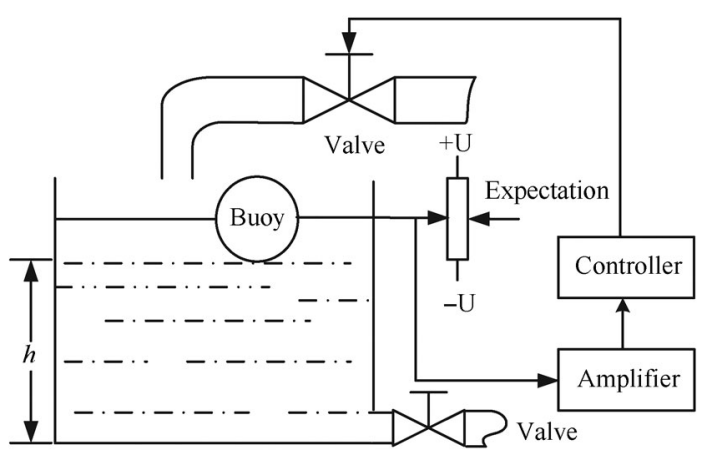

Fig. 2 The water level control system. The amplifier in the simulation experiment is actually a proportional integrator which can be found from "sltank" in Simulink (Version 2009a), and the controller includes GQC, PID, FLC, GQC with PID and GQC with FLC 


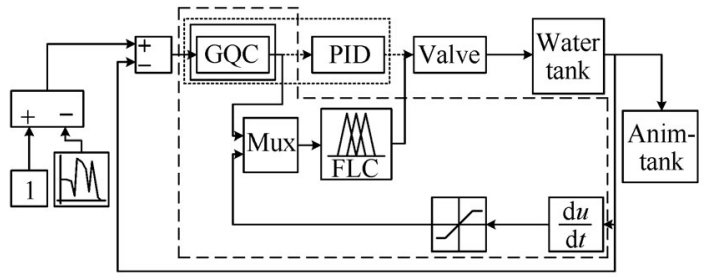

Fig. 3 Simulink model of the system. Parameters of GQC are given later. Details of other blocks can be found from "sltank" in Simulink (Version 2009a). Blocks in the solid line box are used in GQC, blocks in the dotted line box are used in GQC with PID, and these in the dash line box are used in GQC with FLC

Two sets of simulations under Simulink were conducted. In the first set, the expectation of the water level was set to be $1 \mathrm{~m}$, and the expectation was changing around $1 \mathrm{~m}$ with variance 0.01 in the second set. GQC, PID, FLC, GQC with PID, GQC with FLC were used to control the system in both sets. Parameters of GQC, PID and FLC are given below.

1) PID. In the simulations, the PID controller is in the form of " $P+\frac{I}{s}+\frac{D s}{\left(\frac{1}{N s}+1\right)}$ ". We set $P=2, I=0, D=1$ and $N=100 . P, I$ and $D$ are the gains of the corresponding control blocks, and $N$ is the derivative divisor.

2) FLC. The water level error and the change of water level error are taken as inputs to the FLC. The water level error is defined on $[-1,1]$, and the change of water level error is limited on $[-0.1,0.1]$. The output, which indicates the actual water level, is also defined on $[-1,1]$. Membership functions and defuzzication details of the FLC can be found from "sltank" in Simulink (Version 2009a).

3) GQC. In GQC, the subjective uncertain rules are accumulated and integrated to build the grey qualitative matrix. The following are four examples of the subjective uncertain rules, in which $h(t)$ is the water level observed at time $t$ and in $(t+1)$ is the amount of water flows into the tank at $t+1$ :

a) If $h(t) \in[1,+\infty)$, then $i n(t+1)=0$.

b) If $h(t) \in[0,0.25]$, then in $(t+1) \in(0.7,1]$.

c) If $h(t) \in[0,0.5]$, then in $(t+1) \in(0.5,1]$.

d) If $h(t) \in[0,0.75]$, then $\operatorname{in}(t+1) \in(0.3,1]$.

We must proclaim that these subjective uncertain rules are different from fuzzy rules. Unlike the membership functions used in the fuzzy logic to describe the membership of a value to a fuzzy concept, the subjective uncertain rules only state that the input or output belongs to a grey qualitative element, but the actual value is unknown.

To improve the performance, we have to accumulate more subjective rules besides the four rules aforementioned, and integrate them to build the grey qualitative matrix $R$, which is the core of GQC. However, it may not be unique. The dimension and realization of $R$ depend on the cognition capacity. The parameters of GQC we used are as follows.

Input: $p_{0}, p_{1}, \cdots, p_{m}$ and step $s$, which can be constant or variable. For clarity, we set $s$ to be constant. We also set $p_{0}=-1.4, p_{m}=1.4$ and $p_{i}=p_{i-1}+s, i=1, \cdots, m-1$.
Output: $q_{0}, q_{1}, \cdots, q_{n}$, and step $s$. We set $q_{0}=-1.4$, $p_{m}=1.4$, and $q_{j}=q_{j-1}+s, j=1, \cdots, n-1$.

The grey qualitative matrix $R: r_{i j}=1$ if $\otimes_{1}^{i}$ and $\otimes_{2}^{j}$ are related with a subjective uncertain rule, otherwise, $r_{i j}=0$.

The generalized whitening function: For a given $\otimes=$ $[a, b]$, we define $f(a)=a$, and $f(b)=b$. Thus, $\forall p \in \otimes$, the generalized whitening function $f(p)=\lambda_{1} f(a)+\lambda_{2} f(b)=$ $\lambda_{1} a+\lambda_{2} b$. According to the definition, $f(p)$ should satisfy $p=\underset{p \in \otimes}{\arg \max } f(p)$, which is usually the optimal case and hard to achieve. In our paper, we adopt the suboptimal solution, i.e., we select $\lambda_{1}$ (or $\lambda_{2}$ ) uniformly in $[0,1]$, and $\lambda_{1}+\lambda_{2}=1$.

\subsection{Results and analysis}

Fig. 4 shows the results using only GQC. The step $s$ is set to be $0.1 \mathrm{~m}, 0.05 \mathrm{~m}$ and $0.01 \mathrm{~m}$, respectively, which implies that subjective uncertain rules are accumulated and integrated gradually. From Fig. 4 (a), we can conclude that GQC can track the constant expectation level. If the expectation level is changing around $1 \mathrm{~m}$, the tracking results are still good, as shown in Fig. 4 (b). The total tracking errors in $100 \mathrm{~s}$ are $6.700 \mathrm{~m}, 6.638 \mathrm{~m}$, and $6.595 \mathrm{~m}$ for step $0.1 \mathrm{~m}, 0.05 \mathrm{~m}$ and $0.01 \mathrm{~m}$, respectively. However, the results are not good enough due to the cognition limits. Thus, we combine GQC together with PID and FLC separately, and compare the results with traditional PID and FLC. Fig. 5 (a) shows that the overshoot is reduced and the system becomes stable faster by combining GQC with PID than using PID alone. Fig. 5 (b) is the tracking results of GQC with PID and PID alone, the total tracking errors are $6.834 \mathrm{~m}$ for GQC with PID and $7.004 \mathrm{~m}$ for PID. The tracking results are improved. From Fig. 6 (a), we can see that the overshoot of FLC is quite significant, while by combining GQC with FLC, the overshoot is eliminated. Fig. 6 (b) shows that by combining GQC with FLC, the tracking results are also improved, with total tracking errors being $8.092 \mathrm{~m}$ for FLC and $8.058 \mathrm{~m}$ for GQC with FLC, respectively.

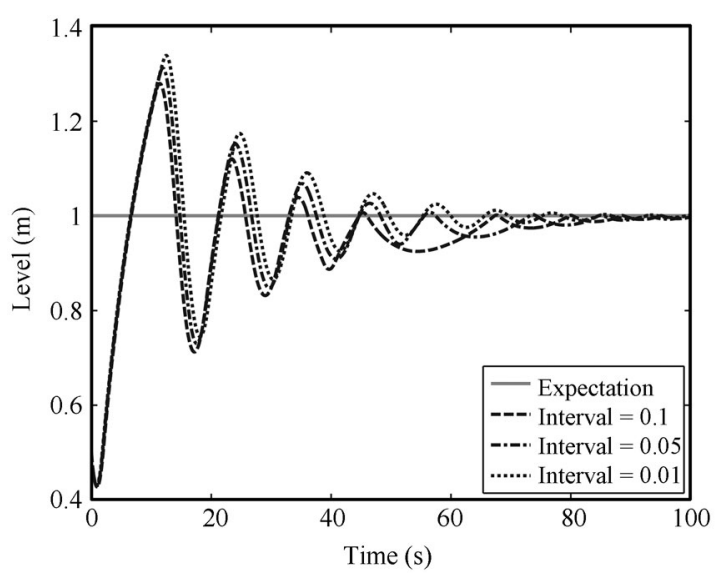

(a) The expectation level is set to $1 \mathrm{~m}$ 


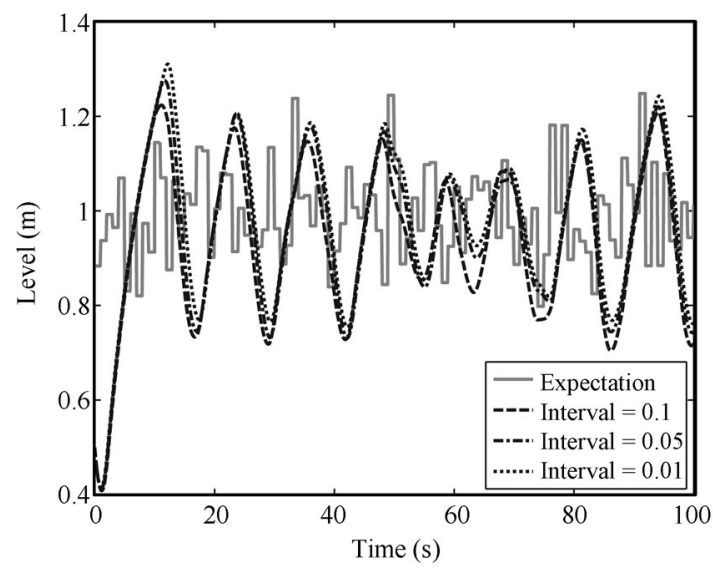

(b) The expectation level is set to change around $1 \mathrm{~m}$

Fig. 5 Results of GQC with different steps

From the simulation results and the theoretical basis, we can conclude that the proposed method could model and control the system well. At the same time, by combining GQC with PID and FLC respectively, their performances are also significantly improved.

\section{Conclusions}

Subjective uncertain rules can be obtained along with the exploration of unknown systems, and they can be used to model and control the systems. However, there is little study on how to represent, accumulate and apply these rules from the perspective of the machine intelligence. In this paper, we propose a novel grey qualitative method for subjective uncertain system modeling and control by combining the grey system theory and the qualitative simulation.

We begin with obtaining subjective uncertain rules and generate the qualitative states, which are represented by the grey qualitative element of the system based on these rules. The grey qualitative matrix is then defined by integrating the subjective uncertain rules gradually. This, on the one hand, improves the description accuracy of the system, on the other hand, avoids the conflicts among different rules.

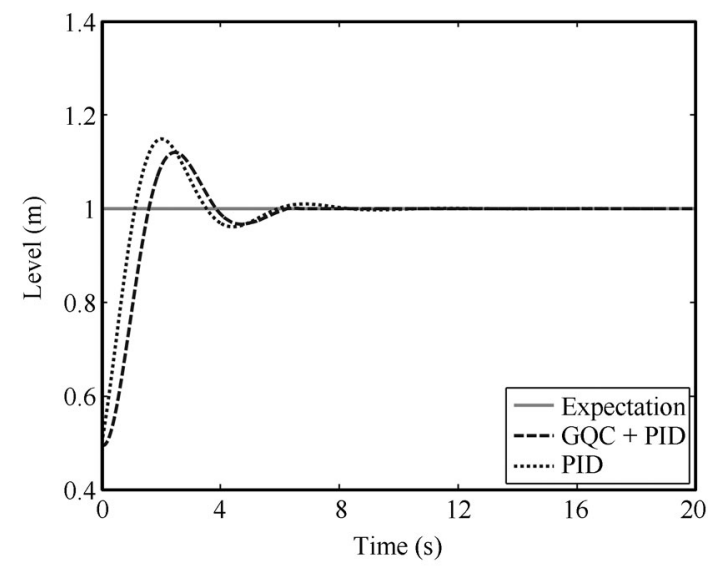

(a) The expectation level is set to $1 \mathrm{~m}$

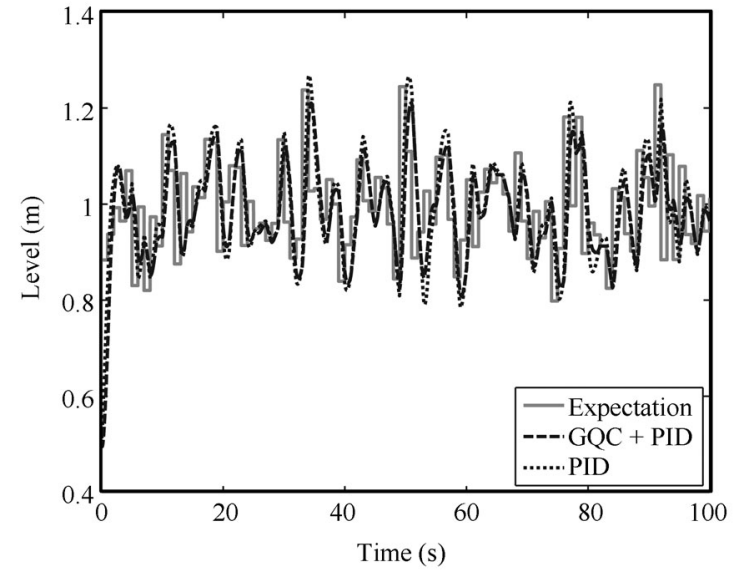

(b) The expectation level is set to change around $1 \mathrm{~m}$ Fig. 5 Comparisons with PID controller

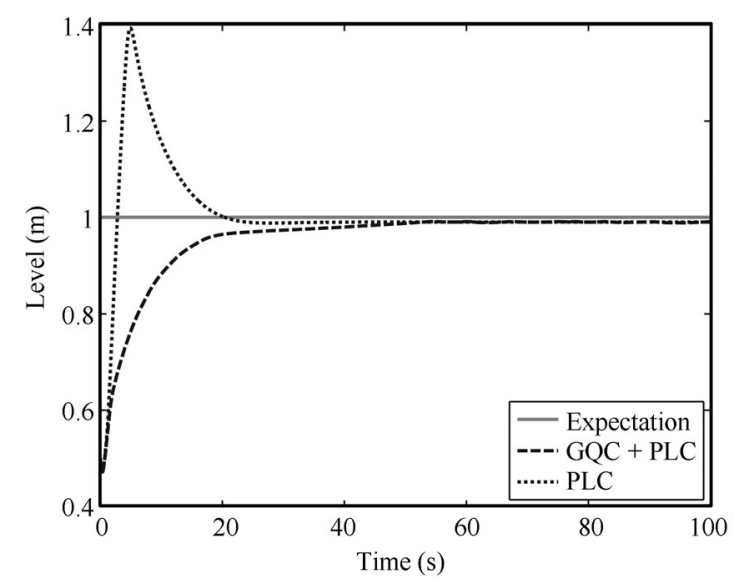

(a) The expectation level is set to $1 \mathrm{~m}$

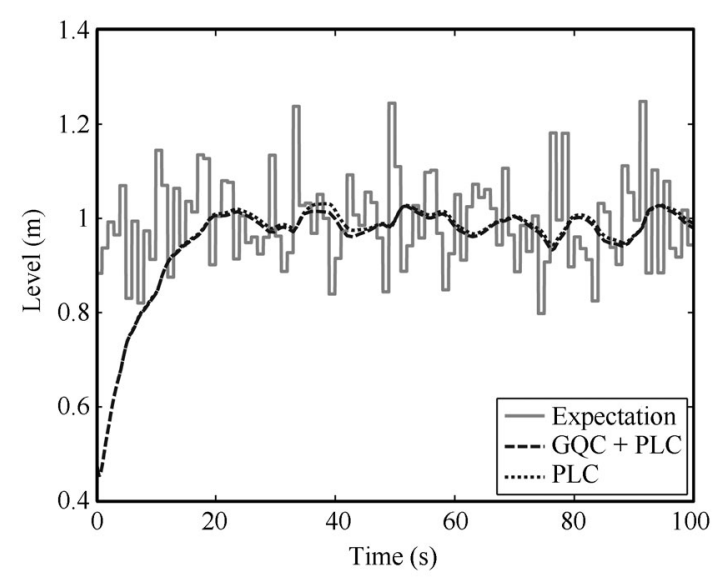

(b) The expectation level is set to change around $1 \mathrm{~m}$

Fig. 6 Comparisons with FLC

Thus, the grey qualitative matrix determines a one-to-one mapping between the input and the output, which are represented by using grey qualitative elements of the system. To obtain the representative value of the grey qualitative element, the generalized whitening function is defined to transform the qualitative information into quantitative 
information. Theoretical analysis, together with simulations compared to PID and FLC, shows that the proposed method can model and control uncertain systems with subjective uncertain rules.

In spite of the effectiveness of our method, it is still a preliminary work of using subjective uncertain rules to model and control systems. The future work will further extend our method both theoretically and experimentally.

\section{References}

[1] L. A. Zadeh. The concept of a linguistic variable and its application to approximate reasoning - I. Information Sciences, vol. 8, no. 3, pp. 199-249, 1975.

[2] L. A. Zadeh. The concept of a linguistic variable and its application to approximate reasoning - II. Information Sciences, vol. 8, no. 4, pp. 301-357, 1975.

[3] L. A. Zadeh. The concept of a linguistic variable and its application to approximate reasoning - III. Information Sciences, vol. 9, no. 1, pp. 43-80, 1975.

[4] L. A. Zadeh. Toward a generalized theory of uncertainty (GTU) - An outline. Information Sciences, vol.172, no. 1, pp. 1-40, 2005.

[5] L. A. Zadeh. Is there a need for fuzzy logic? Information Sciences, vol. 178, no. 13, pp. 2751-2779, 2008.

[6] L. A. Zadeh. Toward extended fuzzy logic-A first step. Fuzzy Sets and Systems, vol.160, no.21, pp.3175-3181, 2009.

[7] B. Kuipers. Qualitative simulation. Artificial Intelligence, vol. 29, no. 3, pp. 289-338, 1986.

[8] B. Kuipers. Qualitative Reasoning: Modeling and Simulation with Incomplete Knowledge, Cambridge, MA: The MIT Press, 1994.

[9] B. Kuipers. Qualitative simulation as causal explanation. IEEE Transactions on Systems, Man and Cybernetics, vol. 17, no. 3, pp. 432-444, 1987.

[10] B. J. Kuipers, C. Chiu, D. T. D. Molle, D. R. Throop. Higher-order derivative constraints in qualitative simulation. Artificial Intelligence, vol. 51, no. 1-3, pp. 343-379, 1991.

[11] D. Berleant, B. J. Kuipers. Qualitative and quantitative simulation: Bridging the gap. Artificial Intelligence, vol. 95, no. 2, pp. 215-255, 1997.

[12] J. L. Deng. Control problems of grey systems. Systems \& Control Letters, vol. 1, no. 5, pp. 288-294, 1982.

[13] H. V. Trivedi, J. K. Singh. Application of grey system theory in the development of a runoff prediction model. Biosystems Engineering, vol. 92, no. 4, pp. 521-526, 2005.

[14] H. X. Li, X. H. Yuan, J. Y. Wang, Y. C. Li. The normal numbers of the fuzzy systems and their classes. Science China Information Sciences, vol. 53, no. 11, pp. 2215-2229, 2010.

[15] H. X. Li. Probability representations of fuzzy systems. Science in China, Series F, vol. 49, no. 3, pp. 339-363, 2006.

[16] J. L. Deng. Introduction to grey system theory. The Journal of Grey System, vol. 1, no. 1, pp. 1-24, 1989.

[17] J. C. Helton. Uncertainty and sensitivity analysis in the presence of stochastic and subjective uncertainty. Journal of Statistical Computation and Simulation, vol. 57, no. 1-4, pp. 3-76, 1997.

[18] Y. L. Huang, Z. H. Chen, W. S. Gui. Grey qualitative simulation. Journal of Grey System, vol.16, no.1, pp. 5-20, 2004.

[19] C. L. Chen, D. Y. Dong, Z. H. Chen, H. B. Wang. Qualitative control for mobile robot navigation based on reinforcement learning and grey system. The Mediterranean Journal of Measurement and Control, vol. 4, no. 1, pp. 1-7, 2008.

记 Springer
[20] C. L. Chen, D. Y. Dong, Z. H. Chen, H. B. Wang. Grey systems for intelligent sensors and information processing. Journal of Systems Engineering and Electronics, vol.19, no. 4, pp. 659-665, 2008.

[21] J. S. Brown, J. De Kleer. The origin, form, and logic of qualitative physical laws. In Proceedings of International Joint Conference on Artificial Intelligence, Karlsruhe, Germany, pp. 1158-1169, 1983.

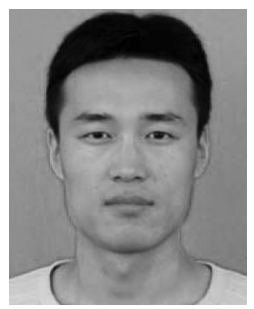

Peng Wang graduated from University of Science and Technology of China (USTC), China in 2010. He is now a Ph. D. candidate at the Laboratory of Simulation and Intelligent Control in the Department of Automation, USTC.

His research interests include system modeling and simulation, mobile robot localization and knowledge representation.

E-mail: pwang@mail.ustc.edu.cn

ORCID iD: 0000-0002-0609-1578

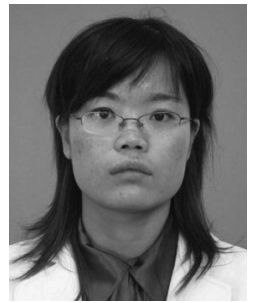

Shu-Jie Li graduated from Hefei University of Technology (HFUT), China in 2004. She received her M. Sc. degree from HFUT in 2008 and her Ph. D. degree from the University of Science and Technology of China (USTC), China in 2012. She is currently a lecturer at the School of Computer and Information, HFUT.

Her research interests include computer aided modeling based on uncertain knowledge.

E-mail: lishujie@mail.ustc.edu.cn

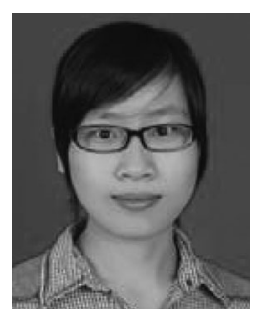

Yan Lv graduated from University of Electronic Science and Technology of China (UESTC), China in 2010. She received her M. Sc. degree from USTC in 2013. She is currently a Ph. D. candidate in Computer Science Department of City University of Hong Kong.

Her research interests include mobile robot localization, path planning and knowledge representation. She is now working on spatial and spatio-temporal databases, big data, and sentiment analysis on social network.

E-mail: lvyanly@mail.ustc.edu.cn

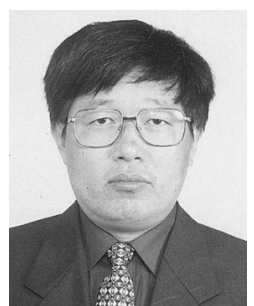

Zong-Hai Chen graduated from University of Science and Technology of China (USTC), China in 1988. He received his M. Sc. degree from USTC in 1991. He is currently a professor at the Laboratory of Simulation and Intelligent Control in the Department of Automation, USTC. He is a member of the Robotics Technical Committee of the International Federation of Automatic Control (IFAC).

His research interests include simulation and optimization control of complex system, theory and technology of intelligent system, and quantum control theory.

E-mail: chenzh@ustc.edu.cn (Corresponding author)

ORCID iD: 0000-0001-9312-9089 